\title{
S stars in the Gaia era: stellar parameters and nucleosynthesis
}

\author{
Sophie Van Eck ${ }^{1}$, Drisya Karinkuzhi ${ }^{1}$, Shreeya Shetye ${ }^{1,2}$, \\ Alain Jorissen ${ }^{1}$, Stéphane Goriely ${ }^{1}$, Lionel Siess ${ }^{1}$, Thibault Merle ${ }^{1}$ \\ and Bertrand Plez ${ }^{3}$ \\ ${ }^{1}$ Institut d'Astronomie et d'Astrophysique, Université Libre de Bruxelles, CP 226, \\ Boulevard du Triomphe, B-1050 Bruxelles, Belgium \\ email: svaneck@ulb.ac.be \\ ${ }^{2}$ Instituut voor Sterrenkunde (IvS), KU Leuven, Celestijnenlaan 200D, \\ B-3001 Leuven, Belgium \\ ${ }^{3}$ LUPM, Université Montpellier 2, CNRS, F-34095 Montpellier, France
}

\begin{abstract}
S stars are s-process and C-enriched $(0.5<\mathrm{C} / \mathrm{O}<1)$ red giants. Their abundances can be determined thanks to a new grid of MARCS model atmospheres covering their whole parameter range. Detailed abundance determinations in intrinsic S stars (TP-AGB) and extrinsic $\mathrm{S}$ stars (binary masqueraders) can provide strong constraints on the s-process nucleosynthesis: in particular, the s-process temperature can be determined using zirconium and niobium abundances, independently of stellar evolution models. Synthetic spectra of dwarf S stars have been computed and will be sought for in spectroscopic survey data, constraining their luminosity thanks to Gaia parallaxes.
\end{abstract}

Keywords. Nuclear reactions, nucleosynthesis, abundances; stars: AGB and post-AGB; stars: atmospheres; stars: abundances.

\section{Introduction}

S-type stars have effective temperatures similar to those of M-type stars, but show prominent $\mathrm{ZrO}$ molecular bands besides the $\mathrm{TiO}$ bands typical of M-type stars. Roughly $50 \%$ of S stars are intrinsic, i.e. thermally-pulsing asymptotic giant branch (TP-AGB) stars experiencing thermal pulses and third dredge-up episodes, while the remaining $50 \%$ are extrinsic and owe their overabundances to a pollution from a binary companion, formerly a TP-AGB star, now an extinct WD. Intrinsic S stars are enriched in technetium, while Tc has completely decayed in extrinsic $\mathrm{S}$ stars $\left(\tau_{1 / 2}\left({ }^{99} \mathrm{Tc}\right)=0.21 \times 10^{6} \mathrm{yrs}\right)$.

\section{S star model atmospheres}

A grid of MARCS model atmospheres (Gustafsson et al., 2008) has been computed for S and SC stars (Van Eck et al. 2017) covering a five-dimensional parameter space: $2700 \mathrm{~K}<\mathrm{Teff}<4000 \mathrm{~K}$ (step $100 \mathrm{~K}$ ); $\log g=0-5$ (step of 1 ), C/O (from 0.5 to 0.99 ); $[\mathrm{s} / \mathrm{Fe}]$ (from 0 to $+2 \mathrm{dex})$, and $[\mathrm{Fe} / \mathrm{H}](0$ or -0.5$)$. Parameters are derived from a comparison between synthetic and observed spectra (Mercator HERMES, Raskin et al. 2011) and photometric colors (Geneva, SAAO). Synthesis of dwarf S star spectra has been attempted (Fig. 1). Given the lack of a currently available LaO linelist, the only prominent differences between dwarf $\mathrm{M}$ and $\mathrm{S}$ stars are (i) the $\mathrm{ZrO}$ bands at $6400 \AA$ and (ii) the $\mathrm{ZrO}$ band at $9300 \AA(\Delta \nu=0)$. The latter, unfortunately, coincides with the $\mathrm{H}_{2} \mathrm{O}$ (201-000) band at $9360 \pm 150 \AA$, and separating the strong telluric component from the weak stellar absorption is by no means easy. Therefore, distinguishing $\mathrm{S}$ and $\mathrm{M}$ dwarfs is not trivial. 

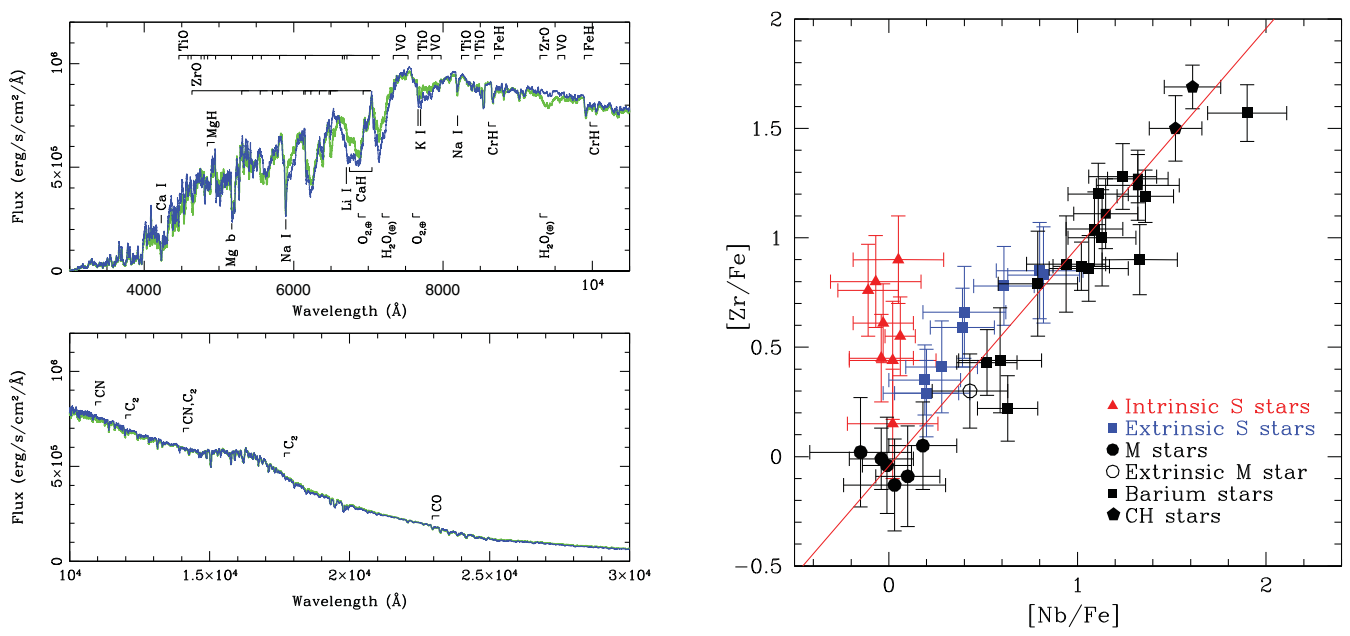

Figure 1. Example of synthetic low-resolution spectra: thick green line: dwarf $\mathrm{S}$ star $(\mathrm{Teff}=3800 \mathrm{~K}, \log \mathrm{g}=4, \mathrm{C} / \mathrm{O}=0.75,[\mathrm{~s} / \mathrm{Fe}]=1)$; thin blue line: dwarf M star (Teff $=3800 \mathrm{~K}, \log$ $\mathrm{g}=4, \mathrm{C} / \mathrm{O}=0.50,[\mathrm{~s} / \mathrm{Fe}]=0)$.

Figure 2. $[\mathrm{Zr} / \mathrm{Fe}]$ and $[\mathrm{Nb} / \mathrm{Fe}]$ for intrinsic and extrinsic S stars, barium stars, non-enriched $\mathrm{M}$ giants (used as reference), and a slightly enriched M giant labelled as 'extrinsic M star'.

\section{Niobium and zirconium abundances: a thermometer}

Since ${ }^{93} \mathrm{Zr}$ decays $\left(\tau_{1 / 2}=1.53 \mathrm{Myr}\right)$ into mono-isotopic $\mathrm{Nb}$, the Nb abundance represents a new powerful diagnostic to separate the families of intrinsic and extrinsic stars, besides the original method based on Tc detection only. Indeed, Fig. 2 is an update of Neyskens et al. (2015) including a new, extended sample of extrinsic stars. It shows that Tc-rich stars (intrinsic S stars) are Nb-poor, while Tc-poor stars (extrinsic S stars and barium stars) are Nb-rich.

For extrinsic stars it can be demonstrated (Neyskens et al. 2015) that, when the abundance of the s-processed material dominates over the initial composition:

$$
\left[\frac{\mathrm{Zr}}{\mathrm{Fe}}\right]=\left[\frac{\mathrm{Nb}}{\mathrm{Fe}}\right]+\log \frac{N_{s}(\mathrm{Zr})}{N_{s}(\mathrm{Nb})}-\log \frac{N_{\odot}(\mathrm{Zr})}{N_{\odot}(\mathrm{Nb})} .
$$

From Fig. 2 it can be seen that, for extrinsic stars, $[\mathrm{Zr} / \mathrm{Fe}]$ as a function of $[\mathrm{Nb} / \mathrm{Fe}]$ follows nicely a straight line of slope 1 (red line on Fig. 2). The y-intercept of this straight line allows to determine $\log \frac{N_{s}(\mathrm{Zr})}{N_{s}(\mathrm{Nb})}$, which is a function of the s-process operation temperature. Therefore $\mathrm{Zr}$ and $\mathrm{Nb}$ abundances in extrinsic stars provide a sensitive sprocess thermometer.

\section{References}

Gustafsson, B., Edvardsson, B., Eriksson, K., Jørgensen, U. G., Nordlund, Å \& Plez, B., 2008, $A \mathscr{E} A, 486,951-970$

Van Eck, S., Neyskens, P., Jorissen, A., Plez, B., Edvardsson, B., Eriksson, K., Gustafsson, B., Jørgensen, U. G., \& Nordlund, A, 2017, A\& $A, 601$, A10

Raskin, G., Van Winckel, H., Hensberge, H., Jorissen, A., Lehmann, H. et al., 2011, A\&\&A, 526, A69

Neyskens, P., Van Eck, S., Jorissen, A., Goriely, S., Siess, L., \& Plez, B., 2015, Nature, 517, 174 